\title{
Mortalidad atribuible al sobrepeso y la obesidad en Argentina: comparación entre los años 2005 y 2009
}

\author{
Laura Débora Acosta* \\ Enrique Peláez ${ }^{\star *}$
}

\begin{abstract}
Este artículo tiene como objetivo comparar la mortalidad atribuible al sobrepeso y la obesidad en personas mayores de 20 años de Argentina, según sexo y grupo de edad, entre los años 2005 y 2009. Para este propósito se utilizaron fuentes de datos secundarias: la Encuesta Nacional de Factores de Riesgo (ENFR) de 2005 y 2009 y estadísticas vitales. Para la estimación de la mortalidad atribuible al sobrepeso y la obesidad se empleó el método dependiente de la prevalencia, basado en el cálculo de la Fracción Atribuible Poblacional (FAP), mientras que para determinar el impacto en la esperanza de vida se calcularon los Años de Esperanza de Vida Perdidos (AEVP). Los principales resultados indican que, en 2005, el 5,2\% de las muertes de varones y el 6,1\% de las de mujeres se debieron al exceso de peso; en 2009 disminuyeron al 4,9\% y al 5,5\%, respectivamente. En 2009 se produjo además un descenso de las muertes por enfermedades cardiovasculares y diabetes en ambos sexos, al tiempo que aumentaron las neoplasias. Los AEVP atribuibles al exceso de peso fueron de 0,33 años en 2005 y 0,30 años en 2009 en el caso de las mujeres, y de 0,45 años en 2005 y 0,43 años en 2009 en el de los varones. Además, se observó un aumento de los AEVP en las edades más jóvenes. Contrariamente a la hipótesis planteada, no se encontró un aumento de la mortalidad atribuible al sobrepeso y la obesidad en el período analizado, aunque se incrementó la mortalidad por esta causa en los más jóvenes.
\end{abstract}

Palabras clave: Mortalidad. Obesidad. Enfermedades crónicas. Esperanza de vida.

\footnotetext{
* Centro de Investigaciones y Estudios sobre Cultura y Sociedad - CIECS, Unidad Ejecutora del Consejo Nacional de Investigaciones Científicas y Técnicas - CONICET y Universidad Nacional de Córdoba, Córdoba, Argentina (Idacosta@conicet.gov.ar).

${ }^{\star *}$ Centro de Investigaciones y Estudios sobre Cultura y Sociedad - CIECS, Unidad Ejecutora del Consejo Nacional de Investigaciones Científicas y Técnicas - CONICET y Universidad Nacional de Córdoba, Argentina (enpelaez@hotmail.com).
} 


\section{Introducción}

Según la Organización Mundial de la Salud (OMS), las prevalencias de sobrepeso y obesidad se están incrementando tanto en los países desarrollados como en aquellos en desarrollo (WHO, 2000). De acuerdo a este organismo, las consecuencias del exceso de peso son numerosas y variadas: desde un aumento del riesgo de muerte prematura hasta complicaciones que afectan la calidad de vida de la persona. El sobrepeso y la obesidad constituyen el principal factor de riesgo para las llamadas enfermedades no transmisibles (ENT), como la diabetes, las enfermedades cardiovasculares y el cáncer, además de estar asociadas a innumerables consecuencias psicológicas (WHO, 2000).

En América Latina, la prevalencia del exceso de peso está aumentando de manera similar a la observada en las sociedades más avanzadas (MONTERO, 2002). Según datos de la OMS del año 2010, los países de la región que presentan mayor proporción de población con obesidad son Chile $(25,7 \%)$ y Uruguay (24,6\%), con valores similares a los registrados en algunos países de Europa (WHO, 2015). En Argentina la población adulta con obesidad representó el 14,6\% en 2005, según la Encuesta Nacional de Factores de Riesgo (ENFR), mientras que en 2009 aumentó significativamente (al 18,0\%) (FERRANTE et al., 2011). Por lo tanto, el aumento de la prevalencia del exceso de peso podría estar implicado en el incremento de la morbilidad y consecuentemente la mortalidad por enfermedades no transmisibles.

En el campo de la epidemiología, el abordaje estándar para estimar los efectos de determinados factores de riesgo - como el exceso de peso- en la morbilidad y mortalidad consiste en el cálculo de la Fracción Atribuible Poblacional (FAP) de la enfermedad o el daño, estimada por medio de la función de la prevalencia de la exposición, y el riesgo relativo (RR) ${ }^{1}$ del grupo expuesto comparado con el grupo no expuesto a ese factor de riesgo (MATHERS et al., 2001).

Para estimar el impacto de la mortalidad por determinadas causas en la esperanza de vida, los índices más utilizados son los Años Potenciales de Vida Perdidos (APVP) y los Años de Vida Saludables, utilizados por la Organización Panamericana de la Salud (OPS, 2003). Estos índices tienen la ventaja de que su cálculo solamente requiere como insumo la información sobre las defunciones por edades y la población total. Sin embargo, están afectados por la estructura etaria (ARRIAGA, 1996), por lo que no serían adecuados para comparar el nivel de mortalidad de las poblaciones.

Por su parte, el método de los Años de Esperanza de Vida Perdidos (AEVP), desarrollado por Arriaga (1996), permite hacer una evaluación cuantitativa de la importancia de las causas de muerte. Este índice trata de determinar cuántos años de vida en promedio

\footnotetext{
${ }_{1}^{1}$ Para establecer si una determinada exposición constituye un factor de riesgo se obtiene el Riesgo Relativo (RR), que se calcula dividiendo la tasa de incidencia en la población expuesta por la población no expuesta. Cuando el resultado es mayor a 1 indica que existe un mayor riesgo de padecer el evento no deseado -en este caso la muerte- para aquella persona que presenta un factor determinado -en este caso el exceso de peso. Cuando es menor a 1 se considera que es un factor de protección, y cuando es igual a 1 indica que no existe relación causal (BONITA et al.; 2008, p. 43).
} 
pierde una población total por la muerte de las personas a determinadas edades. Según el autor: "Con base en las tasas específicas de mortalidad por edades, se calcula la vida promedio que la población vive (esperanza de vida) y se determina cuántos años de vida se pierden considerando la hipótesis sobre cuántos años debería vivir” (ARRIAGA, 1996, p. 9).

Este índice tiene la ventaja de no estar afectado por la estructura de edades de la población. Por otra parte, el indicador se mide en años, por lo que resulta una medida de interpretación sencilla y facilita la comparación entre poblaciones. Lo más destacable de los AEVP es la posibilidad de una fácil interpretación sobre la contribución de cada causa de muerte a la mortalidad total. También permite concentrar los recursos destinados a la salud pública -si existen los medios y es factible- para atacar aquellas causas que más contribuyen a disminuir la esperanza de vida de la población.

La aplicación del concepto de los años de vida perdidos (ARRIAGA, 1996) parte del supuesto de mortalidad nula entre dos edades. Este método no considera de igual forma las defunciones de jóvenes que las de adultos, ya que teóricamente si fallece un joven de 15 años se pierden muchos más años por vivir que si fallece un adulto de 58 años, por ejemplo. Al morir el joven, le resta muchos más años al promedio de vida de la población. El método de los AEVP explica en su totalidad el cambio de las esperanzas de vida, indicando la contribución de cada edad y cada causa de muerte a ese cambio total.

En un estudio reciente realizado en Argentina, Elgart et al. (2010) estimaron la mortalidad atribuible al sobrepeso y la obesidad con los datos de prevalencia de la ENFR de 2005, y determinaron que hubo en total 14.776 muertes debidas a esta causa, con lo que se perdieron 596.704 años de vida saludable.

Sobre la base de estos antecedentes, el presente artículo tiene como objetivo comparar la mortalidad atribuible al sobrepeso y la obesidad en Argentina entre 2005 y 2009 en personas mayores de 20 años, según sexo y grupo de edad. La hipótesis que se propone es que el aumento de la prevalencia del sobrepeso y la obesidad arrojado por la última ENFR (ARGENTINA, MSN, 2011) podría influir en un incremento de la mortalidad atribuible a esta causa en el país.

\section{Metodología}

Para este artículo se utilizaron fuentes de datos secundarias. Las cifras de mortalidad observada se obtuvieron de la Base de Datos de Defunción de la Dirección de Estadísticas e Información en Salud del Ministerio de Salud de la Nación (DEIS). Respecto de la calidad de los datos de las estadísticas de defunción en el país, la Comisión Económica para América Latina y el Caribe (CEPAL, 2010) ha indicado que entre 2005 y 2009 el porcentaje de subregistro de la mortalidad de Argentina fue del $5 \%$ en promedio. Además, existe un $7,5 \%$ de causas mal definidas, otro indicador que informa sobre la calidad de los registros vitales, una proporción baja comparada con la de otros países de Latinoamérica, pero que 
dista de ser la ideal. También continúa siendo elevada la proporción de "códigos basura"2, que alcanzan más del 20\% en la mayoría de las jurisdicciones del país (CEPAL, 2010).

Las causas de muerte fueron categorizadas según la Décima Clasificación Internacional de Enfermedades y Problemas Relacionados con la Salud (CIE-10) (OMS, 1992), calculándose el promedio de defunciones producidas en los años 2004 a 2006 y 2008 a 2010, centrado en los años 2005 y 2009 respectivamente. Además, se utilizaron las bases de datos de la ENFR realizada en 2005 y 2009 por el Instituto Nacional de Estadística y Censos (INDEC) y el Ministerio de Salud de la Nación. El objetivo de esta encuesta fue estimar la prevalencia de los factores de riesgo en la población adulta de Argentina, y determinar el perfil de la población en riesgo mediante sus características demográficas, educativas, socioeconómicas y del entorno familiar y social (ARGENTINA, MSN, 2011).

La población objetivo de esta encuesta fueron las personas de 18 años y más que habitan en hogares de localidades de 5.000 habitantes o más. El muestreo fue probabilístico, polietápico por conglomerados (ARGENTINA, MSN, 2011). La muestra final fue de $n=$ 41.392 en 2005 y de $n=34.732$ en 2009 . Los datos relativos al sobrepeso y la obesidad se obtuvieron por autoinforme del peso y la talla del encuestado. El Ministerio de Salud realizó un estudio de validación del peso y la altura arrojados por la encuesta (ARGENTINA, MSN, 2004), que permitió observar una elevada correlación en el reporte de ambos datos autoinformados y de las mediciones antropométricas - para el peso $(r=0.89$; $p<0.001)$ y para la altura ( $r=0.88$; $p<0.001)$.

Se utilizaron los datos de proyecciones de población elaborados por el INDEC para los años 2005 y 2009 (ARGENTINA, MSN, 2006, 2010). En primer lugar, se determinaron las muertes directamente atribuibles al sobrepeso y la obesidad (Códigos CIE-10 E65-E66) en ambas fechas. Posteriormente, se estimó la mortalidad atribuible a causas relacionadas al sobrepeso y la obesidad (enfermedades cardiovasculares, cáncer y diabetes mellitus tipo II), para lo que se empleó el método dependiente de la prevalencia (FLEGAL et al., 2004), basado en el cálculo de la Fracción Atribuible Poblacional (FAP).

La FAP es un indicador de riesgo poblacional: se refiere a la proporción de la población que está en riesgo de padecer un daño, en relación a su exposición a un determinado factor de riesgo. Para obtener este indicador se requiere conocer la frecuencia del factor de riesgo en la población (BONITA et al., 2008, p. 42). Un factor de riesgo es algún hábito personal o una exposición ambiental que se asocia con un aumento de la probabilidad de que se produzca una enfermedad (BONITA et al., 2008, p. 39). En este caso, el factor de riesgo es el sobrepeso/obesidad, que incrementa la probabilidad del aumento del padecimiento de enfermedades no transmisibles y consecuentemente de morir.

La Ecuación 1 indica el método de cálculo de la FAP, donde $P_{0}$ es la prevalencia de estado nutricional normal, $\mathrm{P}_{1}$ la prevalencia de sobrepeso y $\mathrm{P}_{2}$ la prevalencia de obesidad.

\footnotetext{
${ }^{2}$ Causas definidas pero poco específicas, residuales, terminales, intermedias, que no reflejan la causa básica, por ejemplo, “insuficiencia cardíaca”, o "cáncer”, sin especificar su localización.
} 
El $R_{1}$ es el riesgo relativo para el sobrepeso y el $R_{2}$ el riesgo relativo para la obesidad. Los valores de las prevalencias fueron obtenidos de las ENFR de 2005 y 2009 (Anexo 1).

$\mathrm{FAP}=\frac{\left(P_{0}+P_{1} * R R_{1}+P_{2}{ }^{*} R R_{2}\right)-1}{P_{0}+P_{1}{ }^{*} R R_{1}+P_{2}{ }^{*} R R_{2}}$

Al no disponer de datos de riesgos relativos para la población de Argentina, se siguieron los lineamientos del estudio de "Carga Mundial de la Enfermedad" (GBD por su sigla en inglés), que recomienda el uso de riesgos relativos provenientes de meta-análisis (EZZATI et al., 2006). En consecuencia, las causas de muerte atribuibles al sobrepeso y la obesidad y los correspondientes valores de riesgo relativo fueron obtenidos del meta-análisis realizado por Guh et al. (2009) (Anexo 2).

Una vez obtenida la FAP, se la multiplicó por la mortalidad observada (MO) por cada causa relacionada al exceso de peso, obteniéndose el total de muertes atribuibles al sobrepeso y la obesidad (Ecuación 2):

$\mathrm{MA}=\mathrm{MO}$ * $\mathrm{FAP}$

Se estimó la mortalidad atribuible al sobrepeso y la obesidad según sexo y grupo de edad quinquenal para la población de 20 años y más en 2005 y 2009, suavizando los casos con el promedio de defunciones producidas en los trienios 2004 a 2006 y 2008 a 2010. Se calcularon tasas de mortalidad según edad y sexo expresadas por 10.000 habitantes. Además, se calcularon proporciones de muertes por grandes grupos de causa (diabetes tipo II, enfermedades cardiovasculares y neoplasias) y sexo, con los respectivos intervalos de confianza (IC).

Por último, se calcularon los AEVP mediante el método propuesto por Arriaga (1996). Sobre la base de las tasas específicas de mortalidad por edades se calcularon los años de vida promedio que la población vive (esperanza de vida) entre los 20 y los 80 años, y se determinó cuántos años de vida se pierden considerando la hipótesis de mortalidad nula entre esas edades. El índice AEVP permite medir tanto el nivel de mortalidad de una población como el cambio cuando se comparan dos poblaciones o períodos, y el método de cálculo basado en las tablas de vida posibilita el ajuste por la estructura etaria de la población (ARRIAGA, 1996).

Para el cálculo de la mortalidad atribuible al sobrepeso y la obesidad, y para determinar los AEVP en cada año y la comparación de los dos períodos, se utilizó el programa EPIDAT 4.0 de la Organización Panamericana de la Salud (REVISTA PANAMERICANA DE SALUD PÚBLICA, 2010).

\section{Resultados}

El promedio de muertes de personas de 20 años y más en el período 2004-2006 fue de 146.552 en el caso de los varones y de 131.369 en el de las mujeres. De esas muertes, 7.649 fueron atribuibles a la obesidad en los varones $(5,2 \%)$ y 8.045 en las mujeres 
(6,1\%). En 2009 se produjeron 152.683 muertes de varones y 140.488 de mujeres, de las cuales $7.513(4,9 \%)$ fueron atribuibles a obesidad en el primer caso y 7.772 (5,5\%) en el segundo. En la Tabla 1 se resumen las defunciones totales y aquellas estimadas atribuibles al sobrepeso y la obesidad, según causa, sexo y año. Cabe destacar que, en números absolutos, las muertes totales por las causas relacionadas con el sobrepeso y la obesidad disminuyeron entre 2005 y 2009 , mientras que aquellas producidas directamente por la obesidad aumentaron.

TABLA 1

Muertes totales y muertes atribuibles al sobrepeso y la obesidad en adultos de 20 años y más, por sexo, según causas

Argentina - 2005-2009

\begin{tabular}{|c|c|c|c|c|c|c|c|c|}
\hline \multirow{3}{*}{ Causas de muerte } & \multicolumn{4}{|c|}{2005} & \multicolumn{4}{|c|}{2009} \\
\hline & \multicolumn{2}{|c|}{ Varones } & \multicolumn{2}{|c|}{ Mujeres } & \multicolumn{2}{|c|}{ Varones } & \multicolumn{2}{|c|}{ Mujeres } \\
\hline & MT (1) & $\begin{array}{c}\text { MASO } \\
\text { (2) }\end{array}$ & MT (1) & $\begin{array}{l}\text { MASO } \\
\text { (2) }\end{array}$ & MT (1) & $\begin{array}{l}\text { MASO } \\
(2)\end{array}$ & MT (1) & $\begin{array}{l}\text { MASO } \\
(2)\end{array}$ \\
\hline Diabetes tipo II (E11- E14) & 4.251 & 2.666 & 4.127 & 3.117 & 3.775 & 2.423 & 3.664 & 2.793 \\
\hline $\begin{array}{l}\text { Cáncer de mama (mujeres) } \\
\text { (C50) }\end{array}$ & 35 & - & 5.313 & 273 & 50 & - & 5.357 & 285 \\
\hline Cáncer colorrectal (C18-C21) & 3.279 & 958 & 2.900 & 647 & 3.556 & 1.070 & 3.103 & 707 \\
\hline Cáncer de endometrio (C54.1) & - & - & 362 & 136 & - & - & 344 & 133 \\
\hline Cáncer de esófago (C15) & 1.316 & 122 & 578 & 49 & 1.211 & 115 & 565 & 49 \\
\hline Cáncer de riñón (C64) & 997 & 258 & 478 & 181 & 1.075 & 288 & 521 & 201 \\
\hline Cáncer de ovario (C56) & - & - & 1.092 & 117 & - & - & 1.117 & 125 \\
\hline Cáncer de páncreas (C25) & 1.671 & 454 & 1.808 & 302 & 1.809 & 517 & 1.932 & 333 \\
\hline Cáncer de próstata (C61) & 3.774 & 260 & - & - & 3.827 & 261 & - & - \\
\hline Hipertensión (110-|15) & 2.457 & 530 & 2.913 & 937 & 2.576 & 570 & 3.143 & 1016 \\
\hline $\begin{array}{l}\text { Enfermedad arterial coronaria } \\
(125.0,125.1)\end{array}$ & 825 & 168 & 839 & 321 & 701 & 148 & 612 & 236 \\
\hline $\begin{array}{l}\text { Enfermedad cardíaca } \\
\text { congestiva (150.0) }\end{array}$ & 1.251 & 266 & 1.544 & 281 & 1.174 & 255 & 1.526 & 279 \\
\hline $\begin{array}{l}\text { Accidente cerebrovascular } \\
(160-169)\end{array}$ & 10.110 & 1.675 & 10.662 & 1.298 & 9.167 & 1.562 & 9.740 & 1.211 \\
\hline Embolia pulmonar (126) & 534 & 245 & 751 & 323 & 510 & 241 & 738 & 324 \\
\hline Osteoartritis (M19) & 8 & 3 & 20 & 6 & 9 & 4 & 23 & 6 \\
\hline $\begin{array}{l}\text { Obesidad } \\
\text { Otras causas no relacionadas }\end{array}$ & 43 & 43 & 57 & 57 & 59 & 59 & 74 & 74 \\
\hline con sobrepeso y obesidad & 138.903 & - & 123.324 & - & 145.170 & - & 132.716 & - \\
\hline Total & 146.552 & 7.649 & 131.369 & 8.045 & 152.683 & 7.5131 & 140.488 & 7.772 \\
\hline
\end{tabular}

Fuente: ENFR 2005 y 2009 (INDEC), Estadísticas Vitales 2004-2006 y 2008-2010 (Ministerio de Salud de la Nación), y Guh et al. (2009).

(1) Muertes totales.

(2) Muertes atribuibles al sobrepeso y la obesidad.

Si se agrupan las causas de muerte relacionadas con el exceso de peso en diabetes tipo II, neoplasias y enfermedades cardiovasculares, se observa que la principal causa de muerte relacionada con la obesidad y el sobrepeso la constituyeron las enfermedades cardiovasculares, que en el caso de los varones representaron el 37,7\% de las muertes en 2005 y el 36,9\% en 2009, y entre las mujeres alcanzaron el 39,3\% en 2005 y el 39,4\% en 2009 . 
En ambos sexos, y en los dos años analizados, se observa que la diabetes tipo II constituye la segunda causa de muerte relacionada con la obesidad y el sobrepeso, y la principal causa individual de muerte, ocasionando el 34,8\% y el 32,3\% de las defunciones atribuibles a esta causa en los varones en 2005 y 2009, respectivamente, y en el caso de las mujeres el $38,7 \%$ en 2005 y el $35,9 \%$ en 2009 . Todas las neoplasias constituyeron el $26,8 \%$ de las muertes atribuibles al sobrepeso y la obesidad en los varones en 2005 y el 30,0\% en 2009, mientras que en las mujeres representaron el $21,2 \%$ en 2005 y el 23,6\% en 2009.

La tasa de mortalidad asociada a la obesidad y el sobrepeso para la población total de 20 años y más fue de 6,3 por 10.000 habitantes en los varones y de 6,1 en las mujeres en 2005, mientras que en 2009 fue de 5,9 por 10.000 habitantes en los varones y de 5,6 en las mujeres.

Poniendo el foco en la distribución etaria, se destaca claramente un aumento de la mortalidad relacionada al sobrepeso y la obesidad a medida que aumenta la edad: es menor a una muerte por 10.000 habitantes en los adultos de 20 años y más, y llega a más de 50 muertes por 10.000 en los adultos de 80 años y más, en ambos sexos.

En relación a los cambios producidos entre ambos períodos, en el Gráfico 1 se observan las tasas de mortalidad atribuibles al exceso de peso en escala logarítmica, para una mejor visualización de las diferencias en las edades más jóvenes, en especial en las mujeres. Se aprecia que las tasas de mortalidad relacionadas a esta causa aumentaron en 2009 en las edades más jóvenes de ambos sexos, al tiempo que disminuyeron fundamentalmente a partir de los 40 años. El análisis según sexo muestra que en ambos años los varones registraron una mayor tasa de mortalidad por esta causa que las mujeres en todas las edades (Gráfico 1).

También se calcularon los AEVP entre los 20 y los 80 años. En el caso de las mujeres, en 2005 se perdieron 5,006 años de esperanza de vida entre las edades consideradas, de los cuales 0,33 años fueron atribuibles al sobrepeso y la obesidad (6,6\%). En 2009, en tanto, los AEVP fueron 5,004, de los cuales 0,30 años se perdieron debido al exceso de peso (6,0\%). En el caso de los varones, en tanto, los AEVP totales entre las edades de 20 a 80 años fueron de 9,09 años en 2005; de ellos, 0,45 años fueron atribuibles al sobrepeso y la obesidad (5,0\%). En 2009, por su parte, los AEVP totales de los varones fueron 8,89, y los causados por el sobrepeso y la obesidad 0,43 (4,8\%).

En la Tabla 2 se observan los AEVP por causa de muerte y el cambio relativo anual entre 2005 y 2009 . En el grupo de las mujeres, el cambio relativo anual total fue de -0,0009, es decir que en su caso los AEVP totales disminuyeron en un $0,0009 \%$ por cada año del período de estudio. Los AEVP atribuibles específicamente al sobrepeso y la obesidad disminuyeron un $0,1163 \%$ por cada año; la mayor disminución se observó en la diabetes tipo II, seguida de los accidentes cerebrovasculares y la enfermedad arterial coronaria. Cabe destacar que si bien la mayor reducción se observó en la diabetes tipo II, esta constituyó la principal causa de pérdida de años de vida atribuibles al sobrepeso y la obesidad. Por otra parte, el mayor incremento anual correspondió a la mortalidad 
por obesidad como causa directa, seguida de la mortalidad por cáncer colorrectal y de páncreas ligadas al exceso de peso.

\section{GRÁFICO 1}

Tasas de mortalidad por muertes atribuibles al sobrepeso y la obesidad en adultos de 20 años y más, por sexo, según grupo de edad (escala logarítmica)

Argentina - 2005-2009

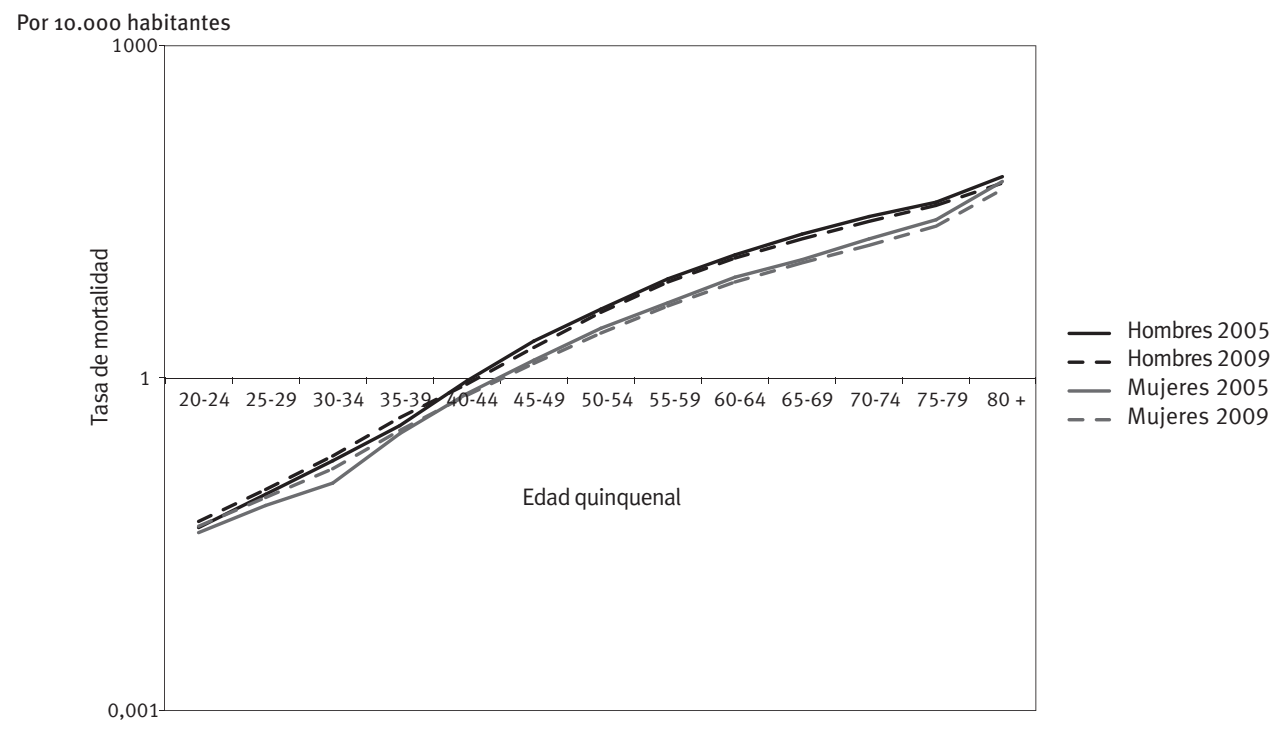

Fuente: ENFR 2005 y 2009, Estadísticas Vitales (MSN, 2004-2006; 2008-2010) y Guh et al. (2009).

Entre los varones se produjo una reducción de los AEVP de 0,036\% entre las edades de 20 y 80 años por cada año del período. Las causas de muerte atribuibles al sobrepeso y la obesidad, en tanto, se redujeron $1,1019 \%$ por cada año del período estudiado. El mayor incremento de AEVP se observó en la mortalidad por obesidad como causa directa, seguida del cáncer colorrectal y el de páncreas, al tiempo que se redujeron la enfermedad cardíaca congestiva, la diabetes tipo II y los accidentes cerebrovasculares. Al igual que en las mujeres, la mayor pérdida de años en ambos períodos fue a causa de la diabetes tipo Il atribuible al exceso de peso (Tabla 2).

Poniendo la mirada analítica en el grupo de edad, se observa un ligero aumento de los AEVP a causa del exceso de peso entre las mujeres de 20 a 39 años entre 2005 y 2009. En el resto de las edades se redujeron, en especial a partir de los 65 años, o se mantuvieron estables. En los varones también se aprecia un leve incremento entre los 20 y los 39 años, mientras que en el resto de las edades disminuyeron hasta los 70 años, momento a partir del cual se mantuvieron estables (Gráfico 2). 
TABLA 2

Comparación de los años de esperanza de vida perdidos (AEVP) atribuibles al sobrepeso y la obesidad en adultos de 20 años y más y cambio relativo anual, por sexo, según causas de muerte Argentina - 2005-2009

\begin{tabular}{|c|c|c|c|c|c|c|}
\hline \multirow[b]{2}{*}{ Causas de muerte } & \multicolumn{3}{|c|}{ Mujeres } & \multicolumn{3}{|c|}{ Hombres } \\
\hline & 2005 & 2009 & $\begin{array}{c}\text { CRA (1) } \\
(\%)\end{array}$ & 2005 & 2009 & $\begin{array}{c}\text { CRA (1) } \\
(\%)\end{array}$ \\
\hline Diabetes tipo II & 0,1376 & 0,1183 & $-0,2195$ & 0,1619 & 0,1403 & $-0,2093$ \\
\hline Cáncer de mama & 0,0187 & 0,0185 & $-0,0142$ & - & - & - \\
\hline Cáncer colorrectal & 0,0304 & 0,0330 & 0,1454 & 0,0591 & 0,0648 & 0,1666 \\
\hline Cáncer de endometrio & 0,0075 & 0,0073 & $-0,0390$ & - & - & - \\
\hline Cáncer de esófago & 0,0018 & 0,0017 & $-0,1233$ & 0,0079 & 0,0074 & $-0,1119$ \\
\hline Cáncer de riñón & 0,0103 & 0,0107 & 0,0688 & 0,0196 & 0,0203 & 0,0578 \\
\hline Cáncer de ovario & 0,008 & 0,0081 & 0,0258 & - & - & - \\
\hline Cáncer de páncreas & 0,0147 & 0,0156 & 0,0993 & 0,0316 & 0,0342 & 0,1458 \\
\hline Cáncer de próstata & & & - & 0,0082 & 0,0077 & $-0,0833$ \\
\hline Hipertensión & 0,0234 & 0,0216 & $-0,1283$ & 0,0281 & 0,0269 & $-0,0723$ \\
\hline Enfermedad arterial coronaria & 0,0045 & 0,0039 & $-0,1920$ & 0,0075 & 0,0070 & $-0,1002$ \\
\hline Enfermedad cardíaca congestiva & 0,0045 & 0,0043 & $-0,1051$ & 0,0101 & 0,0087 & $-0,2212$ \\
\hline Accidente cerebrovascular & 0,0492 & 0,0427 & $-0,2085$ & 0,102 & 0,0884 & $-0,2079$ \\
\hline Embolia pulmonar & 0,0115 & 0,0116 & 0,0102 & 0,0138 & 0,0132 & $-0,0771$ \\
\hline Osteoartritis & 0,0000 & 0,0000 & - & 0,0000 & 0,0000 & - \\
\hline Obesidad & 0,0040 & 0,0055 & 0,8241 & 0,0042 & 0,0063 & 1,1334 \\
\hline Causas no atribuibles al sobrepeso y la obesidad & 4,6800 & 4,701 & 0,0075 & 8,6373 & 8,4675 & $-0,0324$ \\
\hline Total causas atribuibles al sobrepeso y la obesidad & 0,3261 & 0,3028 & $-0,1163$ & 0,454 & 0,4252 & $-0,1019$ \\
\hline Total AEVP & 5,0063 & 5,0037 & $-0,0009$ & 9,0913 & 8,8927 & $-0,0360$ \\
\hline
\end{tabular}

Fuente: ENFR 2005 y 2009 (INDEC), Estadísticas Vitales 2004-2006 y 2008-2010 (Ministerio de Salud de la Nación), y Guh et al. (2009).

(1) Cambio relativo anual.

GRÁFICO 2

Años de esperanza de vida perdidos a causa de la mortalidad atribuible al sobrepeso y la obesidad en adultos de 20 años y más, por sexo, según grupo de edad

Argentina - 2005-2009

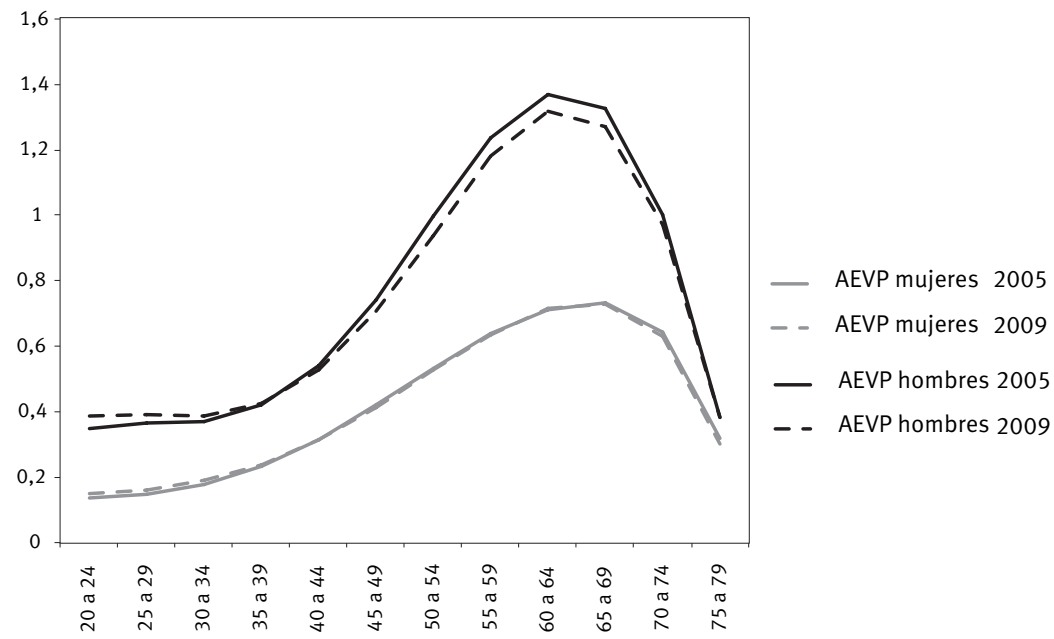

Fuente: ENFR 2005 y 2009, Estadísticas Vitales (MSN, 2004-2006; 2008-2010) y Guh et al. (2009). 


\section{Discusión}

Si bien en el corto período analizado se incrementó la prevalencia del sobrepeso y la obesidad, esto no se tradujo en un aumento del número de muertes atribuibles al exceso de peso, que por el contrario disminuyeron. Además, cabe destacar que entre 2005 y 2009 se produjo una disminución de las muertes relacionadas a esta causa.

Las explicaciones para esta paradoja se encuentran por una parte en la multicausalidad de los procesos de salud-enfermedad (ROTHMAN; GREELAND, 2005), ya que existen numerosas causas que influyen en la prevalencia de estas enfermedades crónicas, además del exceso de peso. Por ejemplo, el consumo de tabaco es un factor de riesgo que interviene fuertemente en la prevalencia de enfermedades no transmisibles como las neoplasias y las enfermedades cardiovasculares. Según Ferrante et al. (2011, p. 39), "el consumo de tabaco y la exposición ambiental al humo de tabaco ajeno disminuyó en forma significativa entre los años 2005 y 2009". De la misma manera, estos autores indican que otro avance que se ha observado en Argentina mediante el análisis de los datos de la ENFR es la mayor medición de la presión arterial, la glucemia y el colesterol (FERRANTE et al., 2011, p. 40), que está relacionada con la prevención secundaria, es decir, con la detección precoz y el tratamiento oportuno de las patologías crónicas (BONITA et al., 2008).

Otra hipótesis que se puede plantear es que al aumentar la obesidad y disminuir la mortalidad pueden haber aumentado la morbilidad y la discapacidad, pero como ha mejorado el tratamiento de estas enfermedades, se produciría la subsecuente sobrevida de la población. Esta constituiría la hipótesis más fuerte, ya que la ENFR muestra que en 2009 se incrementaron por ejemplo la hipercolesterolemia y la diabetes (FERRANTE et al., 2011). Por otra parte, se destaca que la mortalidad por obesidad como causa directa, a pesar que presentó una prevalencia baja en relación a la mortalidad atribuible al exceso de peso como causa subyacente, experimentó el mayor incremento de AEVP en el período analizado, en ambos sexos.

Dentro de las causas de muerte relacionadas con el exceso de peso se produjo una mayor reducción de las defunciones debidas a enfermedades cardiovasculares y diabetes tipo II en ambos sexos, al tiempo que se incrementó la proporción de muertes por neoplasias. Este comportamiento se ha observado en los últimos años en numerosos países desarrollados, como Francia, España, Holanda e Italia (ALLENDER et al., 2008; GIOVANETTI et al., 2011), y puede ser reflejo de un mejoramiento de las condiciones de vida de la población, cambios en la dieta y, fundamentalmente, un mejor tratamiento de las enfermedades cardiovasculares (PALMIERI et al., 2010).

Argentina registra una mortalidad atribuible al sobrepeso y la obesidad similar a la de otros países desarrollados como Francia (5,8\%), menor que la observada en Reino Unido $(8,7 \%)$ (BANEGAS et al., 2003), y muy inferior a la de Estados Unidos (13\%) (ALLISON et al., 1999). Existen pocos datos sobre la mortalidad atribuible al exceso de peso en los países de América Latina. En un estudio sobre carga de mortalidad realizado en Chile se estimó 
que la mortalidad por esta causa fue del 9,1\% del total de las defunciones en 2007, para ambos sexos (UNIVERSIDAD CATÓLICA DE CHILE, 2008).

Los AEVP atribuibles al exceso de peso en Argentina han sido menores que los observados en 11 países desarrollados según el estudio de Preston y Stokes (2011), en el que los valores rondaron entre 1,28 años en las mujeres y 1,68 años en los varones en Estados Unidos hasta 0,50 años en las mujeres y 0,72 años en los varones en Suiza (PRESTON; STOKES, 2011).

Por último, en relación a la distribución por edades, a pesar que se observó una disminución de la mortalidad general, también se ha registrado un leve aumento de la mortalidad en las edades jóvenes en ambos sexos. Esto podría estar asociado a un menor desarrollo de medidas preventivas en la población de este segmento etario, tanto en relación a la atención primaria como a la secundaria (REUSER et al., 2008).

Como conclusión se observa que, contrario a la hipótesis planteada al inicio, a pesar que existió un aumento de la prevalencia del exceso de peso en la población adulta de Argentina, no se produjo un aumento de la mortalidad atribuible al sobrepeso y la obesidad, aunque la mortalidad por obesidad como causa directa aumentó en el período, y se observó un incremento de la pérdida de años de vida atribuibles al exceso de peso en la población joven de ambos sexos.

La principal limitación de este trabajo es que, debido a que en el contexto nacional y en el de América Latina no se han realizado investigaciones que determinen los riesgos relativos de morir atribuibles al exceso de peso, se utilizaron los reportados por un meta-análisis, según las recomendaciones del estudio GBD (EZZATI et al., 2006), tal como se lo ha hecho en otros trabajos (MARTíN-RAMIRO et al., 2014; Kelly et al., 2009). Como los riesgos relativos para una determinada causa de muerte son un valor constante, no explican las diferencias observadas entre los períodos analizados.

Otras limitaciones de este estudio son el corto período examinado y la necesidad de profundizar el estudio mediante un análisis conjunto con otros factores de riesgo asociados a las enfermedades no transmisibles, como por ejemplo el consumo de tabaco, alcohol o la realización de actividad física.

El sobrepeso y la obesidad constituyen dos condiciones en aumento en Argentina, con múltiples consecuencias para el bienestar de la población. A pesar que se registró un incremento de la prevalencia del exceso de peso entre 2005 y 2009 en la población adulta del país - que indicaría que las acciones preventivas para disminuir esta problemática no han sido suficientes-, puede destacarse como aspecto positivo el hecho que el aumento de la prevalencia del sobrepeso y la obesidad no ha contribuido de manera significativa al incremento de la mortalidad, lo que podría estar indicando mejoras en las políticas sociosanitarias que contribuyen con el tratamiento y la detección precoz de las enfermedades no transmisibles.

En los últimos años se ha avanzado en la elaboración de metodologías que aportan al conocimiento de la contribución de los factores de riesgo de las enfermedades no trans- 
misibles a la morbilidad y la mortalidad, su impacto económico, entre otros aspectos. La evaluación del impacto de los factores de riesgo en la mortalidad permite determinar las necesidades y evaluar diferentes aspectos de las políticas de salud. El presente estudio puede completarse evaluando el impacto de otros factores de riesgo sobre la mortalidad en el país - por ejemplo, el consumo de tabaco-y realizando una valoración de riesgos competitivos.

\section{Referencias}

ALLENDER, S.; SCARBOROUGH, P.; PETO, V.; RAYNER, M.; LEAL, J.; LUENGO-FERNANDEZ, R.; GRAY, A. European cardiovascular disease statistics, 2008 edition. Oxford: University of Oxford: Department of Public Health, 2008.

ALLISON, D. B.; FONTAINE, K.; MANSON, J. E. Annual deaths attributable to obesity in United States. JAMA, v. 282, n. 16, p. 1530-1538, 1999.

ARGENTINA. Ministerio de Salud de la Nación. Adaptación transcultural y adaptación de la herramienta para Vigilancia de Enfermedades no Transmisibles en Argentina: Encuesta Nacional de Factores de Riesgo. Boletín Epidemiológico Periódico, edición especial: Vigilancia de Enfermedades no Transmisibles, p. 6-18, 2004.

. Ministerio de Salud de la Nación. Estadísticas vitales. Información básica. Año 2005. Buenos Aires: Ministerio de Salud de la Nación, 2006.

. Ministerio de Salud de la Nación. Estadísticas vitales. Información básica. Año 2009. Buenos Aires: Ministerio de Salud de la Nación, 2010.

. Ministerio de Salud de la Nación. Segunda Encuesta Nacional de Factores de Riesgo 2009. Documento de resultados. Buenos Aires: INDEC, 2011.

ARRIAGA, E. E. Los años de vida perdidos: su utilización para medir el nivel y cambio de la mortalidad. Notas de Población, v. 24, n. 63, p. 7-38, 1996.

BANEGAS, J. R.; LÓPEZ-GARCÍA, E.; GUTIÉRREZ-FISAC, J. L.; GUALLAR-CASTILLÓN, P.; RODRÍGUEZARTALEJO, F. A simple estimate of mortality attributable to excess weight in the European Union. European Journal of Clinical Nutrition, n. 57, p. 201-208, 2003.

BONITA, R.; BEAGLEHOLE, R.; KJELLSTRÖM, T. Epidemiología básica. Publicación Científica n. 629. Washington DC: OPS; 2008.

COMISIÓN ECONÓMICA PARA AMÉRICA LATINA Y EL CARIBE. Taller regional: prácticas para mejorar la calidad de los datos de mortalidad y armonización de indicadores del Milenio 4 y 5 : Dos foros de discusión. Santiago de Chile: CEPAL, 2010. Disponible en: 〈http://www.eclac.cl/ cgi-bin/getProd.asp?xml=/MDG/noticias/paginas/9/39439/P39439.xmlyxsl=/MDG/tpl/p18f.xsl>. Accedido en: 14 enero 2014.

ELGART, J.; PFIRTER, G.; GONZÁLEZ, L.; CAPORALE, J.; CORMILLOT, A.; CHIAPE, M. L. et al. Obesidad en Argentina: epidemiología, morbimortalidad e impacto económico. Revista Argent Salud Pública, v. 1, n. 5, p. 6-12, 2010.

EZZATI, M.; VANDER HOORN, S.; LÓPEZ, A. et al. Comparative quantification of mortality and burden of disease attributable to selected risk factors. En: LÓPEZ, A.; MATHERS, C.; EZZATI, M.; JAMISON, D.; MURRAY, C. Global burden of disease and risk factors. Oxford University Press and World Bank, 2006. 
FERRANTE, D.; LINETZKY, B.; KONFINO, J.; KING, A.; VIRGOLINI, M.; LASPIUR, S. Encuesta Nacional de Factores de Riesgo 2009: evolución de la epidemia de enfermedades crónicas no transmisibles en Argentina. Estudio de corte transversal. Revista Argentina de Salud Pública, v. 2, n. 6, p. 34-41, 2011.

FLEGAL, K. M.; GRAUBARD, B. I.; WILLIAMSON, D. F. Methods of calculating deaths attributable to obesity. American Journal of Epidemiology, v. 160, n. 4, p. 331-338, 2004.

GIOVANNETTI, L.; GORINI, G.; MARTINI, A.; CHELLINI, E.; FORNAI, M. G.; SORSO, B.; SENIORICOSTANTINI, A. Is cancer overtaking cardiovascular diseases as the killer number one in men in Tuscany? Tumori, v. 97, n. 1, p. 14-18, 2011.

GUH, D.; ZHANG, W.; BANSBACK, N.; AMARSI, Z.; BIRMINGHAM, L. et al. The incidente of comorbidities related to obesity and overweigth: a systematic review and meta-analysis. BMC Public Health, n. 9, p. 88-107, 2009.

KELLY, C.; PASHAYAN, N.; MUNISAMY, S.; POWLES J. Mortality attributable to excess adiposity in England and Wales in 2003 and 2015: explorations with a spreadsheets implementation of the Comparative Risk Assessment Methodology. Population Health Metrics, v. 7, p. 11-18, 2009.

MARTIN-RAMIRO, J. J.; ALVAREZ MARTíN, E.; GIL PRIETO, R. Mortalidad atribuible al exceso de peso en España. Medicina Clínica (Barc.), v. 142, n. 12, p. 526-530, 2014.

MATHERS, C. D.; VOS, T.; LOPEZ, A. D.; SALOMON, J.; EZZATI, M. (Ed.). National burden of disease studies: a practical guide. Edition 2.0. Global Program on Evidence for Health Policy. Geneva: World Health Organization, 2001.

MONTERO, J. C. Epidemiología de la obesidad en siete países de América Latina. Formación Continuada en Nutrición y Obesidad, v. 5, n. 6, p. 325-330, 2002.

ORGANIZACIÓN MUNDIAL DE LA SALUD. Clasificación Internacional de las Enfermedades. Décima Edición. Ginebra: Organización Mundial de la Salud, 1992.

ORGANIZACIÓN PANAMERICANA DE LA SALUD. Técnicas para la medición del impacto de la mortalidad: Años potenciales de vida perdidos. Boletín Epidemiológico, v. 24, n. 2, 2003.

PALMIERI, L.; BENNETT, K.; GIAMPAOLI, S.; CAPEWELL, S. Explaining the decrease in coronary heart disease mortality in Italy between 1980 and 2000. American Journal of Public Health, v. 100, n. 4, p. 684-692, 2010.

PRESTON, S. H.; STOKES, A. Contribution to obesity to International differences in life expectancies. American Journal of Public Health, v. 101, p. 2137-2143, 2011.

REUSER, M.; BONNEUX, L.; WILLEKENS, F. The burden of mortality at middle and old age is small. A life table analysis of the US Health and Retirement Survey. European Journal of Epidemiology, v. 23, n. 9, p. 601-607, 2008.

REVISTA PANAMERICANA DE SALUD PÚBLICA (Editorial). El Programa Epidat: Usos y perspectivas, v. 27, n. 1, p. 80-82, 2010.

ROTHMAN, K.; GREELAND, S. Causalidad e inferencia causal en epidemiología. American Journal of Public Health, v. 95, n. 1, p. 8, 2005.

UNIVERSIDAD CATÓLICA DE CHILE. Estudio de carga de enfermedad y carga atribuible, Chile 2007. Informe Final. Santiago de Chile: Escuela de Medicina UCC, 2008.

WHO - World Health Organization. Obesity: preventing and managing the global epidemic. Geneva: WHO, 2000 (WHO Technical Report Series, n. 184).

Global Health Observatory Data Repository. Obesity. WHO: Geneva; 2015. Disponible en: 〈http://apps.who.int/gho/data/node.main.A900A?lang=en〉. Accedido en: 07 abr. 2015. 


\section{Sobre los autores}

Laura Débora Acosta es doctora en Demografía y magíster en Salud Pública. Se desempeña como becaria de investigación en el Centro de Investigaciones y Estudios sobre Cultura y Sociedad - CIECS, Unidad Ejecutora del Consejo Nacional de Investigaciones Científicas y Técnicas - CONICET y Universidad Nacional de Córdoba, Argentina.

Enrique Peláez es doctory magíster en Demografía. Se desempeña como investigador adjunto en el Centro de Investigaciones y Estudios sobre Cultura y Sociedad - CIECS, Unidad Ejecutora del Consejo Nacional de Investigaciones Científicas y Técnicas - CONICET y Universidad Nacional de Córdoba, Argentina.

\section{Dirección de contacto}

\section{Laura Débora Acosta}

Centro de Investigaciones y Estudios sobre Cultura y Sociedad

Calle Rondeau 467

5000 - Córdoba, Argentina

\section{Resumo}

Mortalidade atribuível ao sobrepeso e à obesidade na Argentina: comparação entre 2005 e 2009

Este artigo objetiva comparar a mortalidade atribuível ao sobrepeso e à obesidade na Argentina entre 2005 e 2009, em pessoas com mais de 20 anos, segundo sexo e grupo de idade. Foram utilizadas fontes de dados secundários: Pesquisa Nacional de Fatores de Risco (PNFR 2005 e 2009) e estatísticas vitais. Para a estimação da mortalidade atribuível ao sobrepeso e à obesidade, empregou-se o método dependente da prevalência, fundamentado no cálculo da fração atribuível populacional (FAP). Para determinar o impacto na esperança de vida, foram calculados os anos de esperança de vida perdidos (AEVP). Os principais resultados indicam que, em 2005, 5,2\% das mortes em homens e 6,1\% das mortes em mulheres foram atribuíveis ao excesso de peso. Em 2009, em ambos os sexos observa-se um descenso das mortes por enfermidades cardiovasculares e diabetes, ao mesmo tempo que aumentaram as neoplasias. Os AEVP atribuíveis ao excesso de peso, para as mulheres, foram de 0,33 ano em 2005 e 0,30 ano em 2009 e, para os homens, de 0,45 e 0,43 ano em 2005 e 2009, respectivamente. Por faixa etária, verifica-se um aumento dos AEVP em idades mais jovens. Ao contrário da hipótese formulada, não se constata aumento da mortalidade atribuível ao sobrepeso e à obesidade no período analisado, mas a mortalidade por esta causa incrementa-se entre os jovens.

Palavras-chave: Mortalidade. Obesidade. Enfermidades crônicas. Esperança de vida. 


\begin{abstract}
Mortality attributable to overweight and obesity in Argentina: comparison between 2005 and 2009

The aim of this paper is to compare mortality attributable to overweight and obesity in Argentina between the years 2005 and 2009, in adults ages 20 and older, by sex and age group. Secondary data were used: The last National Risk Factors Survey (NRFS) of 2005 and 2009, and mortality statistics. Mortality attributable to overweight and obesity was estimated using the prevalence dependent method, based on the calculation of the population attributable fraction (PAF). To determine the impact on life expectancy, years of life expectancy lost (YLEL) were calculated. The main results indicate that in $2005,5.2 \%$ of deaths among men and $6.1 \%$ of deaths among women were attributable to overweight and obesity; in 2009, it decreased to $4.9 \%$ and $5.5 \%$, respectively. In 2009, for both sexes, there was a decline in deaths due to cardiovascular diseases and diabetes, while deaths from cancer have increased. YLEL attributable to weight excess were 0.33 years in 2005 and 0.30 years in 2009 for women; and 0.45 years in 2005 and 0.43 years in 2009 for men. By age group, an increase of YLEL attributable to overweight and obesity was found. In contrast to the initial hypothesis, it was not observed an increase in mortality attributable to overweight and obesity between 2005 and 2009 in Argentina, but an increase at among the youth was noted.
\end{abstract}

Keywords: Mortality. Obesity. Chronic Diseases. Life Expectancy.

\title{
Anexo 1
}

Porcentaje de sobrepeso y obesidad, por sexo, según grupo de edad quinquenal Argentina - 2005-2009

\begin{tabular}{|c|c|c|c|c|c|c|c|c|}
\hline \multirow{3}{*}{$\begin{array}{l}\text { Grupo de } \\
\text { edad }\end{array}$} & \multicolumn{4}{|c|}{ Hombres } & \multicolumn{4}{|c|}{ Mujeres } \\
\hline & \multicolumn{2}{|c|}{2005} & \multicolumn{2}{|c|}{2009} & \multicolumn{2}{|c|}{2005} & \multicolumn{2}{|c|}{2009} \\
\hline & Sobrepeso & Obesidad & Sobrepeso & Obesidad & Sobrepeso & Obesidad & Sobrepeso & Obesidad \\
\hline 20 a 24 años & 26,40 & 5,50 & 32,40 & 7,40 & 15,10 & 4,90 & 19,50 & 7,70 \\
\hline 25 a 29 años & 37,10 & 10,70 & 40,60 & 12,70 & 17,60 & 7,70 & 21,30 & 10,40 \\
\hline 30 a 34 años & 46,20 & 16,10 & 46,80 & 18,10 & 24,30 & 10,30 & 24,90 & 13,20 \\
\hline 35 a 39 años & 46,50 & 18,80 & 47,30 & 23,30 & 26,90 & 13,90 & 30,20 & 16,00 \\
\hline 40 a 44 años & 46,80 & 23,30 & 47,50 & 25,10 & 26,60 & 18,70 & 30,20 & 20,70 \\
\hline 45 a 49 años & 49,30 & 23,60 & 50,10 & 26,20 & 31,10 & 19,80 & 34,60 & 20,70 \\
\hline 50 a 54 años & 46,40 & 23,70 & 47,90 & 27,00 & 36,40 & 23,50 & 33,60 & 25,40 \\
\hline 55 a 59 años & 48,70 & 23,40 & 45,70 & 29,20 & 37,10 & 22,00 & 37,40 & 24,10 \\
\hline 60 a 64 años & 52,60 & 21,70 & 48,90 & 26,50 & 37,90 & 23,60 & 36,10 & 27,20 \\
\hline 65 a 69 años & 50,10 & 22,40 & 46,10 & 24,60 & 42,20 & 19,80 & 38,90 & 24,70 \\
\hline 70 a 74 años & 47,10 & 19,60 & 47,50 & 20,80 & 37,40 & 20,80 & 39,10 & 22,40 \\
\hline 75 a 79 años & 45,90 & 14,00 & 47,70 & 17,70 & 43,90 & 15,70 & 41,70 & 18,80 \\
\hline 80 años y más & 47,80 & 11,60 & 45,60 & 11,80 & 36,70 & 14,30 & 37,20 & 13,60 \\
\hline Total & 45,45 & 18,03 & 45,70 & 20,80 & 31,78 & 16,54 & 32,67 & 18,84 \\
\hline
\end{tabular}

Fuente: ENFR 2005 y 2009. 


\section{Anexo 2}

Riesgo relativo ( $95 \%$ IC) de mortalidad en condiciones relacionadas al sobrepeso y la obesidad en la población adulta

\begin{tabular}{|c|c|c|c|c|}
\hline \multirow{2}{*}{ Condición y Código CIE-10 } & \multicolumn{2}{|c|}{ Sobrepeso } & \multicolumn{2}{|c|}{ Obesidad } \\
\hline & Hombres & Mujeres & Hombres & Mujeres \\
\hline Diabetes tipo II (E11- E14) & $\begin{array}{c}2,40 \\
(2,12-2,72)\end{array}$ & $\begin{array}{c}3,92 \\
(3,10-4,97)\end{array}$ & $\begin{array}{c}6,74 \\
(5,55-8,19)\end{array}$ & $\begin{array}{c}12,41 \\
(9,03-17,06)\end{array}$ \\
\hline Cáncer de mama (C50) & - & $\begin{array}{c}1,08 \\
(1,03-1,14)\end{array}$ & - & $\begin{array}{c}1,13 \\
(1,05-1,22)\end{array}$ \\
\hline Cáncer colorrectal (C18-C21) & $\begin{array}{c}1,51 \\
(1,37-1,67)\end{array}$ & $\begin{array}{c}1,45 \\
(1,30-1,62)\end{array}$ & $\begin{array}{c}1,95 \\
(1,59-2,39)\end{array}$ & $\begin{array}{c}1,66 \\
(1,52-1,81)\end{array}$ \\
\hline Cáncer de endometrio (C54.1) & - & $\begin{array}{c}1,53 \\
(1,45-1,61)\end{array}$ & - & $\begin{array}{c}3,22 \\
(2,91-3,56)\end{array}$ \\
\hline Cáncer de esófago (C15) & $\begin{array}{c}1,13 \\
(1,02-1,26)\end{array}$ & $\begin{array}{c}1,15 \\
(0,97-1,36)\end{array}$ & $\begin{array}{c}1,21 \\
(0,97-1,52)\end{array}$ & $\begin{array}{c}1,20 \\
(0,95-1,53)\end{array}$ \\
\hline Cáncer de riñón (C64) & $\begin{array}{c}1,40 \\
(1,31-1,49)\end{array}$ & $\begin{array}{c}1,82 \\
(1,68-1,98)\end{array}$ & $\begin{array}{c}1,82 \\
(1,61-2,05)\end{array}$ & $\begin{array}{c}2,64 \\
(2,39-2,90)\end{array}$ \\
\hline Cáncer de ovario (C56) & - & $\begin{array}{c}1,18 \\
(1,12-1,23)\end{array}$ & - & $\begin{array}{c}1,28 \\
(1,20-1,36)\end{array}$ \\
\hline Cáncer de páncreas (C25) & $\begin{array}{c}1,28 \\
(0,94-1,75)\end{array}$ & $\begin{array}{c}1,24 \\
(0,98-1,56)\end{array}$ & $\begin{array}{c}2,29 \\
(1,65-3,19)\end{array}$ & $\begin{array}{c}1,60 \\
(1,17-2,20)\end{array}$ \\
\hline Cáncer de próstata (C61) & $\begin{array}{c}1,14 \\
(1,00-1,31)\end{array}$ & - & $\begin{array}{c}1,05 \\
(0,85-1,30)\end{array}$ & - \\
\hline Hipertensión (110-I15) & $\begin{array}{c}1,28 \\
(1,10-1,50)\end{array}$ & $\begin{array}{c}1,65 \\
(1,24-2,19)\end{array}$ & $\begin{array}{c}1,84 \\
(1,51-2,24)\end{array}$ & $\begin{array}{c}2,42 \\
(1,59-3,67)\end{array}$ \\
\hline $\begin{array}{l}\text { Enfermedad arterial coronaria } \\
(125.0,125.1)\end{array}$ & $\begin{array}{c}1,29 \\
(1,18-1,41)\end{array}$ & $\begin{array}{c}1,80 \\
(1,64-1,98)\end{array}$ & $\begin{array}{c}1,72 \\
(1,51-1,96)\end{array}$ & $\begin{array}{c}3,10 \\
(2,81-3,43)\end{array}$ \\
\hline Enfermedad cardíaca congestiva (150.0) & $\begin{array}{c}1,31 \\
(0,96-1,79)\end{array}$ & $\begin{array}{c}1,27 \\
(0,68-2,37)\end{array}$ & $\begin{array}{c}1,79 \\
(1,24-2,59)\end{array}$ & $\begin{array}{c}1,78 \\
(1,07-2,95)\end{array}$ \\
\hline Accidente cerebrovascular (160-169) & $\begin{array}{c}1,23 \\
(1,13-1,34)\end{array}$ & $\begin{array}{c}1,15 \\
(1,00-1,32)\end{array}$ & $\begin{array}{c}1,51 \\
(1,33-1,72)\end{array}$ & $\begin{array}{c}1,49 \\
(1,27-1,74)\end{array}$ \\
\hline Embolia pulmonar (126) & $\begin{array}{c}1,91 \\
(1,39-2,64)\end{array}$ & $\begin{array}{c}1,91 \\
(1,39-2,64)\end{array}$ & $\begin{array}{c}3,51 \\
(2,61-4,73)\end{array}$ & $\begin{array}{c}3,51 \\
(2,61-4,73)\end{array}$ \\
\hline Osteoartritis (M19) & $\begin{array}{c}2,76 \\
(2,05-3,70)\end{array}$ & $\begin{array}{c}1,80 \\
(1,75-1,85)\end{array}$ & $\begin{array}{c}4,20 \\
(2,76-6,41)\end{array}$ & $\begin{array}{c}1,96 \\
(1,88-2,04)\end{array}$ \\
\hline
\end{tabular}

Fuente: Guh et al. (2009).

Recebido para publicação em 09/11/2014 Recomendado para publicação em 10/02/2015 Aceito para publicação em 15/07/2015 